\title{
A SEMIDIRECT PRODUCT DECOMPOSITION FOR CERTAIN HOPF ALGEBRAS OVER AN ALGEBRAICALLY CLOSED FIELD
}

\author{
RICHARD K. MOLNAR
}

\begin{abstract}
Let $H$ be a finite dimensional Hopf algebra over an algebraically closed field. We show that if $H$ is commutative and the coradical $H_{0}$ is a sub Hopf algebra, then the canonical inclusion $H_{0} \rightarrow H$ has a Hopf algebra retract; or equivalently, if $H$ is cocommutative and the Jacobson radical $J(H)$ is a Hopf ideal, then the canonical projection $H \rightarrow H / J(H)$ has a Hopf algebra section.
\end{abstract}

For a Hopf algebra $H$ we denote the coradical (i.e. the sum of the simple subcoalgebras of $H$ ) by $H_{0}$, and the Jacobson radical by $J(H)$. If $\pi: H \rightarrow K$ is a surjective (resp. injective) Hopf algebra map we say it splits if there exists a Hopf algebra map $\tau: K \rightarrow H$ with $\pi \circ \tau=I_{K}\left(\right.$ resp. $\left.\tau \circ \pi=I_{H}\right)$. The purpose of this paper is to prove that if $H$ is a finite dimensional Hopf algebra over an algebraically closed field we have the following:

(A) If $H$ is commutative and $H_{0}$ is a sub Hopf algebra, then the canonical inclusion $H_{0} \rightarrow H$ splits as a map of Hopf algebras; or equivalently,

(B) If $H$ is cocommutative and $J(H)$ is a Hopf ideal, then the canonical projection $H \rightarrow H / J(H)$ splits as a map of Hopf algebras.

If follows from the results of [3] that the existence of a Hopf algebra splitting in (A) or (B) induces a semidirect product decomposition of the Hopf algebra $H$, and that such splittings are necessarily unique. For the standard facts about Hopf algebras see [1] or [7]; for splittings and exact sequences see [3].

It is easy to see that (A) and (B) are equivalent, for by finite dimensionality we have $J\left(H^{*}\right)=\left(H_{0}\right)^{\perp}$ and so $H_{0} \cong\left(H^{*} / J\left(H^{*}\right)\right)^{*}$. Thus a splitting in one case induces a splitting in the other by transposing. We shall verify (B). We begin by establishing a special case of (B) which is valid over any field. If $G$ is a group, let $k[G]$ denote the group algebra of $G$ over $k$.

Proposition 1. Let $H=k[G]$ where $G$ is a finite group and $k$ is any field. If $J(H)$ is a Hopf ideal of $H$ then the canonical projection $\pi: H \rightarrow H / J(H)$ splits as a map of Hopf algebras.

PROOF. If the characteristic of $k$ is zero (or is relatively prime to the order

Received by the editors January 20, 1976.

AMS (MOS) subject classifications (1970). Primary 16A24; Secondary 13E10.

Key words and phrases. Hopf algebra, coradical, semidirect product. 
of $G)$ then $J(H)=(0)$ by Maschke's theorem so the result is obvious. So we may assume that the characteristic of $k$ is $p>0$, and that $p$ divides the order of $G$.

Since $\pi$ is a Hopf algebra map, it is easy to verify (see 3.6(a) of [3]) that $N=\operatorname{ker}\left(\left.\pi\right|_{G}\right)$ is a normal subgroup of $G$ and $H / J(H) \cong k[G / N]$, i.e. $J(H)$ is equal to the ideal in $H$ generated by the augmentation ideal of $k[N]$.

Now $k[G / N]$ is semisimple, so $p$ does not divide the order of $G / N$. Thus $N$ must contain all elements of $G$ having order a power of $p$. But if $g \in N$ we have $e-g \in \operatorname{ker}(\pi)=J(H)$ (where $e$ is the identity of $G$ ). Thus $e-g$ is nilpotent, so $0=(e-g)^{p^{\alpha}}=e-g^{p^{\alpha}}$ for some positive integer $\alpha$, i.e. $g$ has order a power of $p$. It follows that $N$ is a normal $p$-Sylow subgroup of $G$.

Now the order of $N$ is a power of $p$ by the above, and is thus relatively prime to the order of $G / N$. By Schur's theorem (10.5 of [2]) there is a group homomorphism $i: G / N \rightarrow G$ which splits the restriction of $\pi$ to $G$, and this group homomorphism induces the desired Hopf algebra splitting.

Lemma 1. Let $K \rightarrow H \rightarrow L$ be an exact sequence of finite dimensional Hopf algebras. Then $H$ is semisimple as an algebra if and only if $K$ and $L$ are semisimple.

Proof. Since everything is finite dimensional it is immediate that the given sequence is exact if and only if the induced sequence $L^{*} \rightarrow H^{*} \rightarrow K^{*}$ is exact. Now the lemma follows from the corresponding theorem with "semisimple" replaced by "cosemisimple" (see 2.20 of [5], or [4]).

In [9] M. Takeuchi proved that a commutative or cocommutative Hopf algebra $H$ is faithfully flat over any sub Hopf algebra $K$.

Lemma 2. Let $H$ be a cocommutative Hopf algebra over a field $k$ and $K$ a sub Hopf algebra. Then $J(H) \cap K \subseteq J(K)$.

Proof. If $m$ is a maximal left ideal of $K$ by faithful flatness we have $\mathrm{m} H \cap K=\mathrm{m}$. The lemma then follows from the fact that the Jacobson radical is the intersection of the maximal left ideals.

In [6] J. B. Sullivan proved that if $H$ is a cocommutative Hopf algebra over an algebraically closed field and $H_{0}$ is spanned by grouplike elements then the inclusion $H_{0} \hookrightarrow H$ splits as a map of Hopf algebras. The following proposition is an easy consequence of Sullivan's theorem.

Proposition 2. Let $H$ be a finite dimensional, irreducible, cocommutative Hopf algebra over an algebraically closed field $k$. If $J(H)$ is a Hopf ideal of $H$ then the canonical projection $H \rightarrow H / J(H)$ splits as a map of Hopf algebras.

Proof. We may assume that the characteristic of $k$ is $p \neq 0$ because in characteristic 0 finite dimensionality implies $H=k$ (see 13.0.1 of [8]). Now $H^{*}$ is local since $H$ is irreducible, and $\left(H^{*}\right)_{0} \cong(H / J(H))^{*}$ is a sub Hopf algebra. We have sep $\left(\left(H^{*}\right)_{0}\right) \subseteq \operatorname{sep}\left(H^{*}\right)=k$ by 3.2 of [7] since $H^{*}$ is local. So $\left(H^{*}\right)_{0}$ is cocommutative by Theorem 4.1 of [7] and hence must be spanned 
by its grouplike elements. But then $\left(H^{*}\right)_{0} \rightarrow H^{*}$ splits by Sullivan's theorem and so $H \rightarrow H / J(H)$ splits by duality.

We are now ready to prove our main result.

THEOREM. If $H$ is a cocommutative, finite dimensional Hopf algebra over an algebraically closed field $k$ and $J(H)$ is a Hopf ideal, then there exists a Hopf algebra map which splits the canonical projection $\pi: H \rightarrow H / J(H)$.

Proof. The proof follows by pasting together the special cases in Propositions 1 and 2 by means of the structure theorem for cocommutative Hopf algebras. We recall (8.15 of [8], or see [4]) that this says $H \cong H^{1} \# k[G]$ (Hopf algebra isomorphism) where $H^{1}$ is the irreducible component containing 1 , and $G$ is a finite group. Note that we may assume the characteristic of $k$ is $p>0$ since otherwise $H^{1}=k$ and, as in Proposition $1, J(H)=(0)$.

Let $L=H / J(H)$ and $\pi: H \rightarrow L$ be the canonical map. Now $L \cong L^{1}$ $\# k[G / N]$ where $N=\operatorname{ker}\left(\left.\pi\right|_{G}\right)$ and $L^{1}=\pi\left(H^{1}\right)$ is the irreducible component of $L$ containing 1. Moreover $L$ is semisimple so $L^{1}$ and $k[G / N]$ are semisimple by Lemma 2 and 3.6(c) of [3].

If we let $\pi_{1}=\left.\pi\right|_{H^{1}}$ and $\pi_{2}=\left.\pi\right|_{k[G]}$, then $\pi=\pi_{1} \sharp \pi_{2}$, and we have

$$
\operatorname{ker}\left(\pi_{1}\right)=H^{1} \cap J(H) \subseteq J\left(H^{1}\right) \subseteq \operatorname{ker}\left(\pi_{1}\right),
$$

the first containment following from Lemma 2 and the second from the fact that $L^{1}=\pi_{1}\left(H^{1}\right)$ is semisimple. A similar argument shows

$$
\operatorname{ker}\left(\pi_{2}\right)=k[G] \cap J(H) \subseteq J(k[G]) \subseteq \operatorname{ker}\left(\pi_{2}\right),
$$

and so we have (Hopf ideals!) $\operatorname{ker}\left(\pi_{1}\right)=J\left(H^{1}\right)$ and $\operatorname{ker}\left(\pi_{2}\right)=J(k[G])$. Thus from Propositions 1 and 2 we have Hopf algebra maps $\tau_{1}$ and $\tau_{2}$ splitting $\pi_{1}$ and $\pi_{2}$ respectively. We have the following commutative diagram:

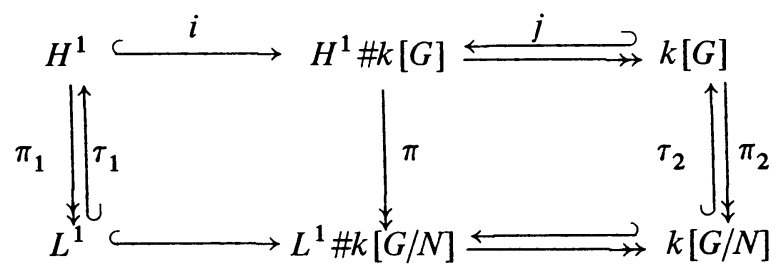

where the horizontal maps are the canonical ones, the rows are exact $(3.6(\mathrm{c})$ of $[3]), \quad \pi_{1} \circ \tau_{1}=I_{L^{1}}, \pi_{2} \circ \tau_{2}=I_{k[G / N]}, H \cong H^{1} \# k[G], \quad$ and $L \cong L^{1}$ $\# k[G / N]$.

Thus we have Hopf algebra maps $i \circ \tau_{1}: L^{1} \rightarrow H$ and $j \circ \tau_{2}: k[G / N] \rightarrow H$ and it is clear from the diagram that $i \circ \tau_{1}$ is a morphism of $k[G / N]$-algebras. So by the universal property of the smash product (1.8 of [1]) there is a map $\tau: L^{1} \# k[G / N] \rightarrow H, \tau=\left(i \circ \tau_{1}\right) \#\left(j \circ \tau_{2}\right)$. This map is clearly a Hopf algebra map (see $\$ 2$ of [3]) and splits $\pi$, so we are done. 


\section{REFERENCES}

1. R. G. Heyneman and M. E. Sweedler, Affine Hopf algebras. I, J. Algebra 13 (1969), 192-241. MR 39 \#6876.

2. S. Mac Lane, Homology, Grundlehren math. Wiss., Band 114, Academic Press, New York; Springer-Verlag, Berlin, 1963. MR 28 \#122.

3. R. K. Molnar, Semi-direct products of Hopf algebras, J. Algebra (to appear).

4. - On the coradical of a Hopf algebra (to appear).

5. J. B. Sullivan, Affine group schemes with integrals, J. Algebra 22 (1972), 546-558. MR 46 \#3553.

6. $\quad$ A decomposition theorem for pro-affine solvable algebraic groups over algebraically closed fields, Amer. J. Math. 95 (1973), 221-228. MR 50 \#13061.

7. M. E. Sweedler, Connected fully reducible affine group schemes in positive characteristic are abelian, J. Math. Kyoto Univ. 11 (1971), 51-70. MR 43 \#6219.

8. - Hopf algebras, Benjamin, New York, 1969. MR 40 \#5705.

9. M. Takeuchi, A correspondence between Hopf ideals and sub Hopf algebras, Manuscripta Math. 7 (1972), 251-270. MR 48 \#328.

Department of Mathematical Sciences, OAKland University, Rochester, Michigan 48063 